\title{
On a Generalized Integral Operator
}

\author{
Irina Dorca ${ }^{1}$, Daniel Breaz ${ }^{2}$, Mugur Acu ${ }^{3}$ \\ ${ }^{1}$ Department of Mathematics, University of Pitesti, Argeş, România \\ ${ }^{2}$ Department of Mathematics, University “1st December 1918” of Alba Iulia, Alba Iulia, România \\ ${ }^{3}$ Department of Mathematics, "Lucian Blaga" University of Sibiu, Sibiu, România \\ Email: irina.dorca@gmail.com
}

Received April 10, 2013; revised May 10, 2013; accepted May 17, 2013

Copyright (C) 2013 Irina Dorca et al. This is an open access article distributed under the Creative Commons Attribution License, which permits unrestricted use, distribution, and reproduction in any medium, provided the original work is properly cited.

\section{ABSTRACT}

We have considered several integral operators from literature and we have made a generalization of them. It can be easily seen that their properties are also preserved. Therefore, we use known results concerning the starlike functions (see $[1,2]$ ) and we unify some known integral operators (see [3]) into one single integral operator, called $I^{1}(z)$, in Section 3 of this paper.

Keywords: Univalent Function; Integral Operator; Generalization; Particular Cases

\section{Introduction}

Let $H(U)$ be the set of functions which are regular in the unit disc $U$,

$$
\mathcal{A}=\left\{f \in \mathcal{H}(U): f(0)=f^{\prime}(0)-1=0\right\}
$$

and

$$
S=\{f \in \mathcal{A}: f \text { is univalent in } U\} .
$$

Prof. G. S. Sălăgean has introduced the subfamily $T$ of $S$ consisting of functions $f$ of the form

$$
f(z)=z-\sum_{j=2}^{\infty} a_{j} z^{j}, a_{j} \geq 0, j=2,3, \cdots, z \in U
$$

in 1999.

Thus, we have the subfamily $S$ - $T$ consisting of functions $f$ of the form

$$
f(z)=z+\sum_{j=2}^{\infty} a_{j} z^{j}, a_{j} \geq 0, j=2,3, \cdots, z \in U
$$

The purpose of this paper is to give a generalization with respect to several integral operators that exist in literature. In order to do so, we have firstly considered the univalent function $f$ and the family $S$ of analytic and univalent functions, notions that we use further.

\section{Preliminary Results}

Remark 2.1. In [1], we have introduced the following operator concerning the functions of Form (1):

$$
D_{\lambda}^{\beta}: A \rightarrow A ; D_{\lambda}^{\beta} f(z)=z-\sum_{j=n+1}^{\infty}[1+(j-1) \lambda]^{\beta} a_{j} z^{j}
$$

Definition 2.1. [2] Let $\beta, \lambda \in R, \beta \geq 0, \lambda \geq 0$ and $f(z)=z+\sum_{j=2}^{\infty} a_{j} z^{j}$. We denote by $D_{\chi}^{\beta}$ the linear operator defined by

$$
\begin{aligned}
& D^{0} f(z)=f(x) ; D^{1} f(z)=D f(z)=z f^{\prime}(z), \\
& D^{n} f(z)=D\left(D^{n-1} f(z)\right)
\end{aligned}
$$

Professor Breaz ([3]) has introduced the following integral operators on univalent function spaces:

$$
\begin{gathered}
J(z)=\left\{\beta \int_{0}^{z}\left[f_{1}^{\prime}\left(t^{n}\right)\right]^{\gamma_{1}} \cdots\left[f_{p}^{\prime}\left(t^{n}\right)\right]^{\gamma_{p}} \mathrm{~d} t\right\}^{\frac{1}{\beta}}, \\
H(z)=\left\{\beta \int_{0}^{z} t^{\beta-1}\left[f_{1}^{\prime}(t)\right]^{\gamma_{1}} \cdots\left[f_{p}^{\prime}(t)\right]^{\gamma_{p}} \mathrm{~d} t\right\}^{\frac{1}{\beta}}, \\
F(z)=\int_{0}^{z}\left(\frac{f_{1}(t)}{t}\right)^{\gamma_{1}} \cdots\left(\frac{f_{p}(t)}{t}\right)^{\gamma_{p}} \mathrm{~d} t, \\
G(z)=\left[\beta \int_{0}^{z}\left(\frac{f_{1}(t)}{t}\right)^{\gamma_{1}} \cdots\left(\frac{f_{p}(t)}{t}\right)^{\gamma_{p}} \mathrm{~d} t\right]^{\frac{1}{\beta}},
\end{gathered}
$$




$$
F_{\gamma, \beta}(z)=\left\{\beta \int_{0}^{z} t^{\beta-1}\left(\frac{f_{1}(t)}{t}\right)^{\frac{1}{\gamma_{1}}} \cdots\left(\frac{f_{p}(t)}{t}\right)^{\frac{1}{\gamma_{p}}} \mathrm{~d} t\right\}^{\frac{1}{\beta}}
$$

and

$$
G_{\gamma, p}(z)=\left\{[p(\gamma-1)+1] \int_{0}^{z} g_{1}^{\gamma-1}(t) \cdots g_{p}^{\gamma-1}(t) \mathrm{d} t\right\}^{\frac{1}{p(\gamma-1)+1}},
$$

where

$$
\gamma_{i}, \gamma, \beta \in \mathbb{C}, \forall i=\overline{1, p}, p \in \mathbb{N}-\{0\}, n \in \mathbb{N}-\{0,1\} .
$$

Let $D^{n}$ be the Sălăgean differential operator, $D^{n}: \mathcal{A} \rightarrow \mathcal{A}, n \in \mathbb{N}$, defined as:

$$
\begin{aligned}
& D^{0} f(z)=f(z), D^{1} f(z)=D f(z)=z f^{\prime}(z), \\
& D^{n} f(z)=D\left(D^{n-1} f(z)\right)
\end{aligned}
$$

and $D^{k}, D^{k}: \mathcal{A} \rightarrow \mathcal{A}, k \in \mathbb{N} \bigcup\{0\}$ of form:

$$
\begin{aligned}
& D^{0} f(z)=f(z), \cdots, \\
& D^{k} f(z)=D\left(D^{k-1} f(z)\right)=z+\sum_{n=2}^{\infty} n^{k} a_{n} z^{n} .
\end{aligned}
$$

The following integral operator is studied in [4], where $f_{i}, i=1, \cdots, n, n \in \mathbb{N}$, are considered to be of Form (2):

Definition 2.2. [4] We define the general integral operator $I_{k, n, \lambda, \mu}: \mathcal{A}_{n} \rightarrow \mathcal{A}$ by

$$
\begin{gathered}
I_{k, n, \lambda, \mu}\left(f_{1}, \cdots, f_{n}\right)=F \\
D^{k} F(z)=\int_{0}^{z}\left(\frac{D_{1}^{\lambda} f_{1}(t)}{t}\right)^{\mu_{1}} \cdots\left(\frac{D_{n}^{\lambda} f_{n}(t)}{t}\right)^{\mu_{n}} \mathrm{~d} t
\end{gathered}
$$

where

$$
\begin{aligned}
& f_{i} \in \mathcal{A}, i \in \mathbb{N}-\{0\}, \lambda=\left(\lambda_{1}, \cdots, \lambda_{n}\right) \in \mathbb{N}_{0}^{n}, \\
& \mu=\left(\mu_{1}, \cdots, \mu_{n}\right) \in \mathbb{N}^{n}, n \in \mathbb{N}
\end{aligned}
$$

and $k \in \mathbb{N}_{0}$.

Lemma 2.1. [5] (Schwarz Lemma) Let the function $f(z)$ be regular in $U$, with $f(0)=0$. If $|f(z)| \leq 1(z \in U)$, then $|f(z)| \leq|z|$ for all $z \in \# U$, where the equality holds only if $f(z)=K z,|K|=1$.

Some integral operators and the related properties of them are also studied in [6,7].

The neighborhoods of the class of functions defined using the operator (3) is studied in [8].

Remark 2.2. In [9], we have introduced and studied the following operator of the functions $f \in \# S$, $S=\{f \in A: f$ is univalent in $U\}:$

$$
\begin{aligned}
& D_{\lambda_{1}, \lambda_{2}}^{n, \beta} f(z)=\left(h * \psi_{1} * f\right)(z) \\
= & z \pm \sum_{k \geq 2} \frac{\left[1-\lambda_{1}(k-1)\right]^{\beta-1}}{\left[1-\lambda_{2}(k-1)\right]^{\beta}} \cdot \frac{1+c}{k+c} \cdot C(n, k) \cdot a_{k} \cdot z^{k}
\end{aligned}
$$

where $C(n, k)=\frac{(n+1)_{k-1}}{(1)_{k-1}} ; \quad(n)_{k}$ is the Pochammer symbol; $k \geq 2, c \geq 0$ and $\operatorname{Re}\{c\} \geq 0 ; z \in \# U$.

In [10], Prof. G. S. Sălăgean has introduced the subfamily $T$ of $S$ consisting of functions $f$ of the Form (1); see Section 1.

Remark 2.3. If we denote by $(x)_{k}$ the Pochammer symbol, we define it as follows:

$$
\begin{aligned}
& (x)_{k} \\
= & \begin{cases}1 & \text { for } k=0, x \in \mathbb{C} \backslash\{0\} \\
x(x+1)(x+2) \cdots(x+k-1) & \text { for } k \in \mathbb{N}-\{0\} \text { and } x \in \mathbb{C} .\end{cases}
\end{aligned}
$$

Following, we introduce a new general integral operator in Theorem 3.1, $I^{1}(z)$, giving also several examples which prove its relevance. We derive it as well in order to show its applicability.

Considering the univalent functions with negative coefficients (see [11]), we develop, $I^{2}(z)$ from Corollary 3.1 .

\section{Main Results}

Theorem 3.1. Let $\alpha, \gamma_{1}, \gamma_{2}, \beta \in \mathbb{C}, \operatorname{Re} \alpha=a>0$ and

$$
\begin{gathered}
D_{\lambda_{1}, \lambda_{2}}^{n, \kappa} f_{j}(z) \in \mathcal{A}, \lambda_{1}, \lambda_{2}, \kappa \geq 0, \sigma \in \mathbb{R}, j=\overline{1, p}, \\
p \in \mathbb{N} ; D_{\lambda_{1}, \lambda_{2}}^{n, \kappa} f_{j}\left(z^{n}\right) \\
\text { of Form (14). If }\left|\frac{\left(D_{\lambda_{1}, \lambda_{2}}^{n, \kappa} f_{j}\left(z^{n}\right)\right)^{\prime \prime}}{\left(D_{\lambda_{1}, \lambda_{2}}^{n, \kappa} f_{j}\left(z^{n}\right)\right)^{\prime}}\right| \leq \frac{1}{n} \text { and } \\
\left|\frac{\left(D_{\lambda_{1}, \lambda_{2}}^{n, \kappa} f_{j}\left(z^{n}\right)\right)^{\prime}}{\left(D_{\lambda_{1}, \lambda_{2}}^{n, \kappa} f_{j}\left(z^{n}\right)\right)}\right| \leq \frac{1}{n}, \forall z \in U, j=\overline{1, p}, \\
\frac{\sum_{j=1}^{p}\left[\left|\delta_{j}^{1}\right| \cdot\left(\left|2 \gamma_{1}-1\right|-|\sigma|\right)+\left|\delta_{j}^{2}\right| \cdot\left(\left|2 \gamma_{2}-1\right|-|\sigma|\right)\right]}{\left|\sigma \cdot\left(2 \gamma_{1}-1\right) \cdot\left(2 \gamma_{2}-1\right) \cdot\left(\prod_{j=1}^{p} \delta_{j}^{1} \cdot \delta_{j}^{2}\right)\right|} \leq 1
\end{gathered}
$$

and

$$
\begin{aligned}
& \left|\sigma \cdot\left(2 \gamma_{1}-1\right) \cdot\left(2 \gamma_{2}-1\right) \cdot\left(\prod_{j=1}^{p} \delta_{j}^{1} \cdot \delta_{j}^{2}\right)\right| \\
& \leq \frac{n+2 a}{2} \cdot\left(\frac{n+2 a}{n}\right)^{\frac{1}{n+2 a}}
\end{aligned}
$$


Then, for

$\forall \delta, \delta_{j}^{1}, \delta_{j}^{2} \in \mathbb{C}, j=1, \cdots, p, \operatorname{Re}(\beta) \geq a, \operatorname{Re}(\beta \delta) \geq a$, the function

$$
\begin{aligned}
I^{1}(z)= & \left\{\beta \int_{0}^{z} t^{\beta \delta-1} \cdot \prod_{j=1}^{p}\left[\frac{\left(D_{\lambda_{1}, \lambda_{2}}^{n, \kappa} f_{j}\left(t^{n}\right)^{\prime}\right)^{2 \gamma_{1}-1}}{t^{\sigma}}\right]^{\delta_{j}^{1}}\right. \\
& \left.\cdot\left[\frac{\left(D_{\lambda_{1}, \lambda_{2}}^{n, \kappa} f_{j}\left(t^{n}\right)\right)^{2 \gamma_{2}-1}}{t^{\sigma}}\right]^{\delta_{j}^{2}} \mathrm{~d} t\right\}^{\frac{1}{\beta}}
\end{aligned}
$$

is univalent for all $n \in \mathbb{N}-\{0\}$.

\section{Proof.}

Let

$$
\begin{aligned}
& h(z) \\
= & \int_{0}^{z} \prod_{j=1}^{p}\left[\frac{\left(D_{\lambda_{1}, \lambda_{2}}^{n, \kappa} f_{j}\left(t^{n}\right)^{\prime}\right)^{2 \gamma_{1}-1}}{t^{\sigma}}\right]^{\delta_{j}^{1}} \cdot\left[\frac{\left(D_{\lambda_{1}, \lambda_{2}}^{n, \kappa} f_{j}\left(t^{n}\right)\right)^{2 \gamma_{2}-1}}{t^{\sigma}}\right]^{\delta_{j}^{2}} \mathrm{~d} t \\
= & \int_{0}^{z} \prod_{j=1}^{p}\left[h_{j}^{1}\left(t^{n}\right)\right]^{\delta_{j}^{1}} \cdot\left[h_{j}^{2}\left(t^{n}\right)\right]^{\delta_{j}^{2}},
\end{aligned}
$$

from where we obtain the following derivatives:

$$
\begin{aligned}
h^{\prime}(z)= & \prod_{j=1}^{p}\left[h_{j}^{1}\left(z^{n}\right)\right]^{\delta_{j}^{1}} \cdot\left[h_{j}^{2}\left(z^{n}\right)\right]^{\delta_{j}^{2}}, \\
h^{\prime \prime}(z)= & \sum_{j=1}^{p} \delta_{j}^{a} \cdot\left(n \cdot z^{n-1}\right) \cdot\left[h_{j}^{a}\left(z^{n}\right)\right]^{\delta_{j}^{a}-1} \\
& \cdot\left[h_{j}^{a}\left(z^{n}\right)\right]^{\prime} \cdot \prod_{l=1, l \neq j}^{p}\left[h_{l}^{b}\left(z^{n}\right)\right]^{\delta_{l}^{b}},
\end{aligned}
$$

$a, b \in\{1,2\}, a \neq b$.

Furthermore, we have that

$$
\begin{gathered}
\frac{h^{\prime \prime}(z)}{h^{\prime}(z)}=\sum_{j=1}^{p} \frac{\left[\left(h_{j}^{a}\left(z^{n}\right)\right)^{\delta_{j}^{a}}\right]^{\prime}}{\left(h_{j}^{a}\left(z^{n}\right)\right)^{\delta_{j}^{a}}}, a \in\{1,2\} \text {, which means } \\
\frac{z h^{\prime \prime}(z)}{h^{\prime}(z)}=\sum_{j=1}^{p}\left\{\delta _ { j } ^ { 1 } \cdot \left[\begin{array}{c}
\left.\left(2 \gamma_{1}-1\right) \cdot \frac{\left(D_{\lambda_{1}, \lambda_{2}}^{n, \kappa} f_{j}\left(t^{n}\right)^{\prime}\right)^{\prime}}{D_{\lambda_{1}, \lambda_{2}}^{n, \kappa} f_{j}\left(t^{n}\right)^{\prime}}-\sigma\right] \\
\left.+\delta_{j}^{2} \cdot\left[\left(2 \gamma_{2}-1\right) \cdot \frac{D_{\lambda_{1}, \lambda_{2}}^{n, \kappa} f_{j}\left(t^{n}\right)^{\prime}}{D_{\lambda_{1}, \lambda_{2}}^{n, \kappa} f_{j}\left(t^{n}\right)}-\sigma\right]\right\}
\end{array}\right.\right.
\end{gathered}
$$

$a \in\{1,2\}$. We consider

$$
p(z)=\frac{1}{\left|\sigma \cdot\left(2 \gamma_{1}-1\right) \cdot\left(2 \gamma_{2}-1\right) \cdot\left(\prod_{j=1}^{p} \delta_{j}^{1} \cdot \delta_{j}^{2}\right)\right|} \cdot \frac{h^{\prime \prime}(z)}{h^{\prime}(z)}
$$

and we obtain

$$
\begin{aligned}
|p(z)| & =\left|\frac{1}{\mid \sigma \cdot\left(2 \gamma_{1}-1\right) \cdot\left(2 \gamma_{2}-1\right) \cdot\left(\prod_{j=1}^{p} \delta_{j}^{1} \cdot \delta_{j}^{2}\right)} \cdot \frac{h^{\prime \prime}(z)}{h^{\prime}(z)}\right| \\
& \leq \frac{\sum_{j=1}^{p}\left[\left|\delta_{j}^{1}\right| \cdot\left(\left|2 \gamma_{1}-1\right|-|\sigma|\right)+\left|\delta_{j}^{2}\right| \cdot\left(\left|2 \gamma_{2}-1\right|-|\sigma|\right)\right]}{\left|\sigma \cdot\left(2 \gamma_{1}-1\right) \cdot\left(2 \gamma_{2}-1\right) \cdot\left(\prod_{j=1}^{p} \delta_{j}^{1} \cdot \delta_{j}^{2}\right)\right|} .
\end{aligned}
$$

Using (16) from hypothesis, we see that $|P(z)| \leq 1$.

By applying Schwarz Lemma, we have that

$\frac{1}{\left|\sigma \cdot\left(2 \gamma_{1}-1\right) \cdot\left(2 \gamma_{2}-1\right) \cdot\left(\prod_{j=1}^{p} \delta_{j}^{1} \cdot \delta_{j}^{2}\right)\right|} \cdot\left|\frac{h^{\prime \prime}(z)}{h^{\prime}(z)}\right| \leq|z|^{n-1} \leq|z|$.

If we multiply the last inequality with $\left(\frac{1-|z|^{2 a}}{a}\right)$, we obtain:

$$
\begin{aligned}
& \left(\frac{1-|z|^{2 a}}{a}\right) \cdot\left|\frac{z h^{\prime \prime}(z)}{h^{\prime}(z)}\right| \\
& \leq\left|\sigma \cdot\left(2 \gamma_{1}-1\right) \cdot\left(2 \gamma_{2}-1\right) \cdot\left(\prod_{j=1}^{p} \delta_{j}^{1} \cdot \delta_{j}^{2}\right)\right| \cdot\left(\frac{1-|z|^{2 a}}{a}\right) \cdot|z|^{n} .
\end{aligned}
$$

Let $Q:[0,1] \rightarrow \mathbb{R}, Q(x)=\frac{1-x^{2 a}}{a} \cdot x^{n}, x=|z|$. We can easily see that $Q(0)=Q(1)=0, Q(x) \geq 0, \forall x \in[0,1]$, and $Q\left(\left(\frac{n}{n+2 a}\right)^{\frac{1}{2 a}}\right)>0$, where $\left(\frac{n}{n+2 a}\right)^{\frac{1}{2 a}}$ is the point of maximum for the function $Q$.

Thus,

$$
\begin{aligned}
& Q(x) \leq Q\left(\left[\frac{n}{n+2 a}\right]^{\frac{1}{2 a}}\right)=\frac{2}{n+2 a} \cdot\left(\frac{n}{n+2 a}\right)^{\frac{n}{2 a}}, \\
& \forall x \in[0,1] .
\end{aligned}
$$

By taking into account the inequalities (17), (19) and (20), we obtain the following: 
$\left(\frac{1-|z|^{2 a}}{a}\right) \cdot\left|\frac{z h^{\prime \prime}(z)}{h^{\prime}(z)}\right| \leq 1$, which means that the function $I^{1}(z)$ of Form (18) is univalent for all $n \in \mathbb{N}-\{0\}$.

Remark 3.1. The mapping properties with respect to integral operator $I^{1}(z)$ of Form (18) are studied in [7].

If we consider the operator $D^{\lambda}(z)$ of Form (3) we obtain the following Corollary. It can be proved in a similar way as the Theorem 3.1 is.

Corollary 3.1. Let $\alpha, \gamma_{1}, \gamma_{2}, \beta \in \mathbb{C}, \operatorname{Re} \alpha=a>0$ and $D_{\lambda}^{\beta} f_{j} z \in \mathcal{A}, \beta \geq 0, \lambda \geq 0, \sigma \in \mathbb{R}, D_{\lambda}^{\beta} f(z)$ of Form (3). If

$$
\begin{gathered}
\left|\frac{\left(D_{\lambda}^{\beta} f_{j}\left(z^{n}\right)\right)^{\prime \prime}}{\left(D_{\lambda}^{\beta} f_{j}\left(z^{n}\right)\right)^{\prime}}\right| \leq \frac{1}{n} \text { and }\left|\frac{\left(D_{\lambda}^{\beta} f_{j}\left(z^{n}\right)\right)^{\prime}}{\left(D_{\lambda}^{\beta} f_{j}\left(z^{n}\right)\right)}\right| \leq \frac{1}{n}, \\
\forall z \in U, j=\overline{1, p}, \\
\frac{\left(\sum_{j=1}^{p}\left[\left|\delta_{j}^{1}\right| \cdot\left(\left|2 \gamma_{1}-1\right|-|\sigma|\right)+\left|\delta_{j}^{2}\right| \cdot\left(\left|2 \gamma_{2}-1\right|-|\sigma|\right)\right]\right)}{\left|\sigma \cdot\left(2 \gamma_{1}-1\right) \cdot\left(2 \gamma_{2}-1\right) \cdot\left(\prod_{j=1}^{p} \delta_{j}^{1} \cdot \delta_{j}^{2}\right)\right|} \leq 1
\end{gathered}
$$

and

$$
\begin{aligned}
& \left|\sigma \cdot\left(2 \gamma_{1}-1\right) \cdot\left(2 \gamma_{2}-1\right) \cdot\left(\prod_{j=1}^{p} \delta_{j}^{1} \cdot \delta_{j}^{2}\right)\right| \\
\leq & \frac{n+2 a}{2} \cdot\left(\frac{n+2 a}{n}\right)^{\frac{1}{n+2 a}},
\end{aligned}
$$

then for all

$$
\delta, \delta_{j}^{1}, \delta_{j}^{2} \in \mathbb{C}, j=1, \cdots, p, \operatorname{Re}(\chi) \geq a, \operatorname{Re}(\chi \delta) \geq a,
$$

the function

$$
\begin{aligned}
I^{2}(z)= & \left\{\chi \int_{0}^{z} t^{\chi \delta-1} \prod_{j=1}^{p}\left[\frac{\left(D_{\lambda}^{\beta} f_{j}\left(t^{n}\right)^{\prime}\right)^{2 \gamma_{1}-1}}{t^{\sigma}}\right]^{\delta_{j}^{1}}\right. \\
& \left.\cdot\left[\frac{\left(D_{\lambda}^{\beta} f_{j}\left(t^{n}\right)\right)^{2 \gamma_{2}-1}}{t^{\sigma}}\right]^{\delta_{j}^{2}} \mathrm{~d} t\right\}^{\frac{1}{\chi}}
\end{aligned}
$$

is univalent for all $n \in \mathbb{N}-\{0\}$.

Remark 3.2. The Corollary is true also for $D^{\lambda}(z)$ of Form (3), $\forall j \in \overline{1, p}, p \in N$.

Next we give few examples of particular cases which can be found in literature.

Let $\beta=0$ in $D^{\lambda}(z)$ of Form (3) or (4). So we have that $D_{\lambda}^{0} f(z)=f(z), \forall \lambda \geq 0$. We will use this form of the integral operator, where the function $f$ is of Form (2) with respect to the operator (21). For further simplification, we consider that $\gamma_{1}=\gamma_{2}=1$ and $\delta=1$ (except of Example 3.5).

For the first four examples we consider

$$
\delta_{j}^{1}=0 j=\overline{1, p}, p \in \mathbb{Z}-\{0\}, n=1 .
$$

Example 3.1. If $\sigma=1, \chi=1$ and we use the notation $\delta_{j}^{2}=\gamma_{j}, j=\overline{1, p}, p \in \mathbb{N}-\{0\}$ we obtain the operator $F(z)$ of Form (7).

Example 3.2. If $\sigma=1$ we obtain the operator $G(z)$ of Form (8) for $\delta_{j}^{2}=1 / \gamma_{j}, j=\overline{1, p}, p \in \mathbb{N}-\{0\}$.

Example 3.3. If $\sigma=1$ and we use the notation $\delta_{j}^{2}=1 / \gamma_{j}, j=\overline{1, p}, p \in \mathbb{N}-\{0\}$ we obtain the operator $F_{\gamma, \chi}(z)$ of Form (9).

Example 3.4. If $\sigma=0$ we obtain the operator $G_{\gamma, p}(z)$ of Form (10) for $\chi=[p(\gamma-1)+1]$ and

$\delta_{j}^{2}=\gamma-1, \delta=\frac{1}{\chi}$.

For the next two examples we consider $\delta_{j}^{2}=0, j=\overline{1, p}, p \in \mathbb{N}-\{0\}$ and $\sigma=0$.

Example 3.5. a) If $\chi=1, \delta=1$, we obtain a particular case of the function $J(z)$ of Form (5), in which $\beta=1, \forall n \in \mathbb{N}-\{0\}$.

b) If $\delta=\frac{1}{\chi}, \delta_{j}^{1}=\gamma_{j}, j=\overline{1, p}, p \in \mathbb{N}-\{0\}$ we obtain the operator $J(z)$ of Form (5).

Example 3.6. If $n=1$ we obtain the operator $H(z)$ of Form (6) for $\delta_{j}^{1}=\gamma_{j}, j=\overline{1, p}, p \in \mathbb{N}-\{0\}$.

Remark 3.3. It can be easily proved that the examples above are also true for $f(z)$ of Form (1).

\section{Conclusions}

The integral operator which is introduced and studied in Theorem 3.1 of this paper, called $I^{1}(z)$, is a generalization of several integral operators that are taken from literature (for e.g., see [3]). Looking to the examples from above, we can easily see that our integral operator can be used further without any concern, because it preserves the initial state of the already known integral operators. Therefore, the integral operator $I^{1}(z)$ covers the integral operators from [3] without loss of generality.

Open Problem 3.1. It would be interesting to prove that the integral operator (18) or (21) covers also the integral operator from [5].

\section{Acknowledgements}

This work was partially supported by the strategic project POSDRU 107/1.5/S/77265, inside POSDRU Romania 2007-2013 co-financed by the European Social FundInvesting in People. 


\section{REFERENCES}

[1] M. Acu, I. Dorca and S. Owa, "On Some Starlike Functions with Negative Coefficients," Proceedings of the International Conference on Theory and Applications of Mathematics and Informatics, Alba Iulia, 21-24 July 2011, pp. 101-112.

[2] M. Acu and S. Owa, "Note on a Class of Starlike Functions," Proceeding of the International Short Joint Work on Study on Calculus Operators in Univalent Function Theory, Kyoto, 2006, pp. 1-10.

[3] D. Breaz, "Integral Operators on Univalent Function Spaces," Academiei Române, Bucureşti, 2004.

[4] D. Breaz, H. O. Güney and G. Ş. Sălăgean, "A New General Integral Operator," Tamsui Oxford Journal of Mathematical Sciences, Vol. 25, No. 4, 2009, pp. 407-414.

[5] M. Darus and R. W. Ibrahim, "Generalized Cesáro Integral Operator," International Journal of Pure and Applied Mathematics, Vol. 69, No. 4, 2011, pp. 421-427.

[6] I. Rahovean (Dorca) and A. I. Rahovean, "New Integral
Operators-Properties," LAP Publishing, Saarbrücken, 2013.

[7] I. Dorca, D. Breaz and M. Acu, "Mapping Properties of Some Classes of Analytic Functions under Generalized Integral Operators," Advances in Mathematics: Scientific Journal, Vol. 1, No. 1, 2012, pp. 51-57.

[8] I. Dorca, M. Acu and D. Breaz, "Note on Neighborhoods of Some Classes of Analytic Functions with Negative Coefficients," ISRN Mathematical Analysis, 2011, Article ID: 610549 .

[9] I. Dorca, D. Breaz and M. Acu, "Subordonation of Certain Subclass of Convex Function," Studia Universitatis Babeş-Bolyai, Vol. 57, No. 2, 2012, pp. 181-187.

[10] G. S. Sălăgean, “Geometria Planului Complex,” Promedia Plus, Cluj-Napoca, 1999.

[11] H. Silverman, "Univalent Functions with Negative Coefficients," Proceedings of the American Mathematical Society, Vol. 5, 1975, pp. 109-116.

http://dx.doi.org/10.1090/S0002-9939-1975-0369678-0 